\title{
Use of Drones for Secure Mobile-Commerce: A Secure Commerce Platform Provides Target Information using Drones and CCTV
}

\author{
Il-Kyu Ha \\ Assistant Professor, Department of Computer Engineering, Kyungil University, \\ Gyeongsan, Gyeongbuk, Rep. of Korea \\ ikha@kiu.kr
}

\begin{abstract}
Recently, drones are used in various fields such as environment, military, and health. This study is an attempt to expand the use of drones while applying drones to the financial sector. The possibility of fraud always exists in direct dealings among individuals. Online trading among individuals provides trading advantages in terms of time, space, and cost. However, this form of trading is often not secure, because of the anonymity of the parties involved during non-face-to-face transactions. Therefore, we often select the direct dealing method. However, even if we opt for the direct dealing method where parties meet offline to enhance stability in the transaction of goods, we are still unable to determine what type of person the other party is or determine their location. Thus, we can be easily exposed to deceit. Therefore, in this research, to reduce the risks involved in direct dealing among individuals, a secure platform is designed and implemented using drones and closed-circuit television (CCTV) streaming and the location information of the trading parties to guarantee safe trading. In particular, the platform provides fast target location information that can be monitored by a manager using drones and pre-installed CCTVs in a designated place with the intent of providing the location information of the seller and the buyer so that they can share mutual trust during the trade.
\end{abstract}

Keywords: Drone, Location Information, Direct Dealing Platform, Online Trading System, Direct Dealing among Individuals, Streaming Service

\section{Introduction}

Today, people trade many goods using Internet online trading, e.g., customer to customer (C2C). However, although Internet trading provides trading advantages in terms of time, space, and cost, in many cases, it is an unsafe trading practice because of the anonymity of the individuals involved, i.e., the identity of the user is not revealed, and the transactions are non-face-to-face. According to the "2016 Internet Usage Survey" conducted by the Future Creative Science Division and the Korea Internet Promotion Agency between July to October 2016, 39.9\% of individuals in online trading were inconvenienced and suffered damages [1].

In addition, even if the parties select the direct trading method in which they meet offline, the said report [1] stated that cases in which their communication is discontinued without notice comprised approximately $6 \%$ of all trades. Similarly, direct trading can be trusted by buyers because the items can be personally examined and a decision to purchase or not can be made on the spot. However, neither trading party will be able to identify what type of person the other party is, or his location. Thus, either party can be easily exposed to fraud [2].

Meanwhile, as the information age has rapidly advanced, online books have been made widely available to the public. However, college students who need handwritten review

Received (December 11, 2017), Review Result (March 8, 2018), Accepted (March 12, 2018) 
materials still use many offline books. The inflation rate last year (2016) increased by $0.7 \%$, whereas the cost of paper books increased by $3.4 \%$ [3]. The consumption patterns of books used by college student have changed owing to the price increases. In other words, instead of buying a new book, students often buy from an online second-hand bookstore or community. In addition, traders who live in the same area prefer direct offline trading for trading convenience and stability in the transaction.

Recently, drones have been utilized for various purposes in various fields. In particular, drones are widely used for public purposes such as rescue of victims, monitoring of disasters and traffic, and for leisure activities in various forms [4-6]. Figure 1 shows the use of drones for various purposes.

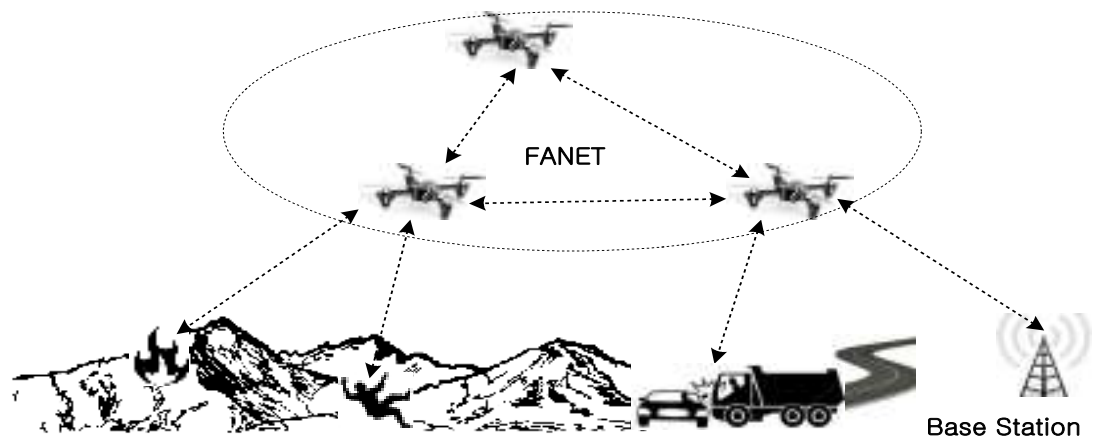

Figure 1. Use of Drones for Various Purposes

In the present study, we propose a secure trading platform that enhances the safety of trading by the fusion of drones and CCTV and provides transaction-location information so that managers can monitor a trading location. In particular, in the proposed platform, traders can meet on an online trading system beforehand and decide to conclude a transaction offline. The parties can move to a safe place, e.g., in a crime prevention through environmental design (CPTED) [7] area, for direct dealing. The trading video stream taken by the CCTV and drones is sent to a manager for monitoring purposes. The position information is provided quickly to the traders and manager to both monitor and identify the other party.

In the following sections, the related research is discussed, the proposed platform is introduced, the implementation procedure of the proposed platform is explained, and finally the conclusions from this study are summarized.

\section{Related Works}

In this section, related works are reviewed and related techniques are discussed.

\subsection{Previous Research Studies}

Many research activities related to improving the stability of direct trading among individuals have been conducted. A summary of the representative works is listed in Table 1. Reference [8] proposed a system that can mediate secure transactions using the Universal Subscriber Identity Module (USIM) of a smartphone. Reference [9] proposed a system that improves security using the International Standard Book Number (ISBN) information of books as a trade item. Reference [10] developed an application for secondhand book trading within a university, and [11] proposed a method of using a one-time password (OTP) for stable trading in a mobile environment. Such studies suffer from problems in that they cannot provide foolproof methods to ensure safe transactions among individuals, such as third-party monitoring of the transaction. Therefore, in the current research, we attempt to develop a system that solves such problems. 


\section{Table 1. Previous Research Works Related to Improvement of Reliability in Direct Trading among Individuals}

\begin{tabular}{|c|c|c|c|}
\hline Study & Contents & $\begin{array}{l}\text { Method for enhancement } \\
\text { of security }\end{array}$ & Problems \\
\hline$[8]$ & $\begin{array}{l}\text { Transaction brokerage system } \\
\text { to prevent third-party fraud in } \\
\mathrm{C} 2 \mathrm{C} \text { e-commerce environment }\end{array}$ & $\begin{array}{l}\text { USIM authentication of } \\
\text { smartphone }\end{array}$ & $\begin{array}{l}\text { Cannot be monitored } \\
\text { by the manager }\end{array}$ \\
\hline [9] & $\begin{array}{l}\text { C2C mobile used-book trading } \\
\text { system }\end{array}$ & $\begin{array}{l}\text { Using ISBN of the used } \\
\text { book }\end{array}$ & $\begin{array}{l}\text { Insufficient trading } \\
\text { security }\end{array}$ \\
\hline$[10]$ & $\begin{array}{l}\text { Smartphone application for } \\
\text { trading of used books }\end{array}$ & $\begin{array}{c}\text { Supports simple } \\
\text { transaction function }\end{array}$ & $\begin{array}{l}\text { Insufficient trading } \\
\text { security }\end{array}$ \\
\hline [11] & $\begin{array}{c}\text { Secure OTP authentication in } \\
\text { mobile environments }\end{array}$ & Using OTP authentication & $\begin{array}{l}\text { Insufficient direct } \\
\text { trading security }\end{array}$ \\
\hline
\end{tabular}

\subsection{Streaming Service}

Streaming is a method in which a supplier provides multimedia data such as music and moving pictures to a user via the Internet, where data are reproduced in real time. There are essentially three techniques for streaming implementation, namely, compression techniques, network technology, and quality-of-service (QoS) technology. QoS technology is not considered in the initial streaming technology but is added for critical quality improvement in the wireless-network service environment. Traditional streaming protocols include real-time streaming protocol (RTSP), real-time transport protocol (RTP), real-time messaging protocol (RTMP), etc. However, these methods have high initial costs and suffer from some drawbacks associated with firewall-protected environments. Figure 2 shows the compression techniques and streaming technologies for a streaming service.

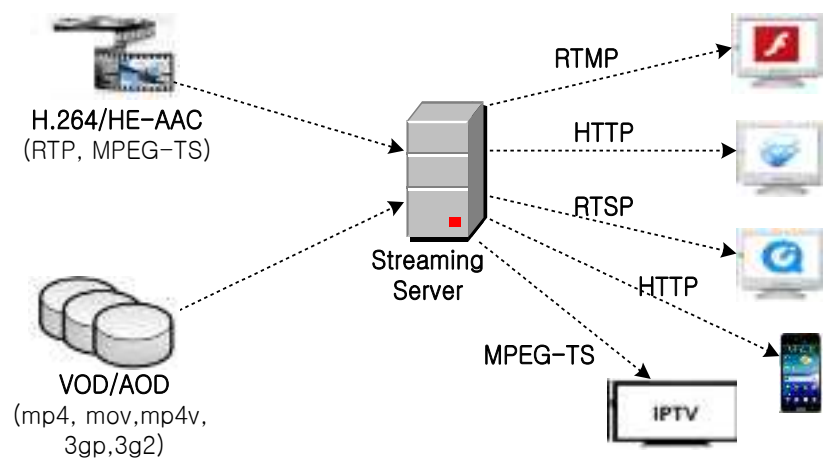

Figure 2. Techniques for a Streaming Service

One method that can solve these drawbacks is the Hypertext Transfer Protocol (HTTP) live streaming (HLS), which implements streaming using HTTP provided by Apple Corporation. H.264, MPEG-TS, and systems in the Codec technology are available that compress and decrypt data for reproduction, e.g., MP4, MOV, and similar formats, in a container format that supports streaming of moving images.

\subsection{Positioning Methods}

We need to learn the location of a trader to provide the location information of the parties in relation to the location of the transaction. The positioning method can be classified into outdoor and indoor positioning. Outdoor positioning mainly uses the Global Positioning System (GPS). In GPS, a GPS satellite that orbits the Earth transmits a radio wave to a receiver. However, the radio waves at an arbitrary reception point contain 
many errors. The causes of the errors are many, such as satellite orbit error, ionosphere delay, convective layer delay, and receiver noise. Research has been conducted on differential GPS to correct the ionosphere-delay error (which has the greatest influence among all errors) [12].

A typical method of indoor positioning is wireless fidelity (Wi-Fi), which is mainly used for building a wireless local area network infrastructure. Wi-Fi also contains errors because the signal transmitted from an access point reaches the receiver via multiple paths with different transmission lengths. Even if it is measured in one place, irregular fading (received signal-strength indication) occurs. Research is under way to estimate true position by correcting this error [13].

\subsection{Utilization of Drones for Target Detection}

As mentioned above, drones can be utilized for various purposes [14-17]. Target detection technology is a fundamental technology necessary for various drone applications. In this research, it is applicable to drones to quickly determine a trader's position information in direct trading. Various studies on existing target detection have been published [18-20]. Reference [18] explains a method in which a drone moves toward a target based on the probability that a target exists, in a situation where the position of the target cannot be known. The study in [19] introduces various autonomous decision algorithms for drone target detection, and [20] introduces a target search method developed from the partially observable Markov decision process (POMDP). In this research, we present only the idea for utilizing drones to determine the exact position of the traders in the designated trading space and to observe the behavior of the traders. The proposed system in this study determines the positions of the traders by accessing the position information through their personal mobile devices.

\section{Design of the Proposed Platform}

In this section, the function and the architecture and development environment of the proposed platform are discussed.

\subsection{Function of the Proposed Platform}

The structure of the proposed secure platform for direct trading among individuals is shown in Figure 3. Trading among individuals is initiated by the online part of the trading platform. After establishing transactions by matching the sales and purchase orders of the buyers and sellers, for quick dealing, the buyers and sellers move to the CPTED location for offline direct trading. Here, the offline part of the proposed platform is activated. It sends videos taken by a CCTV to the streaming server to improve the safety of the transaction, and the manager can monitor and record the trading transaction. The platform sends the position of the traders for monitoring, and the buyer or seller identifies the position of the other based on the position information.

The proposed platform is developed in the form of an application (App) for convenience of use. To conduct secure direct trading among individuals, an App with the following functions is developed:

First, it has a secure login function. To increase safety, a user can only subscribe by logging in after a member registration process and receipt of an e-mail authentication of the organization (university) domain.

Second, it has a secure trading function. The seller posts a trade item (e.g., book), and the buyer inquires and makes a reservation for the transaction subject to the seller's decision. It additionally provides a chat window for communication between parties. 


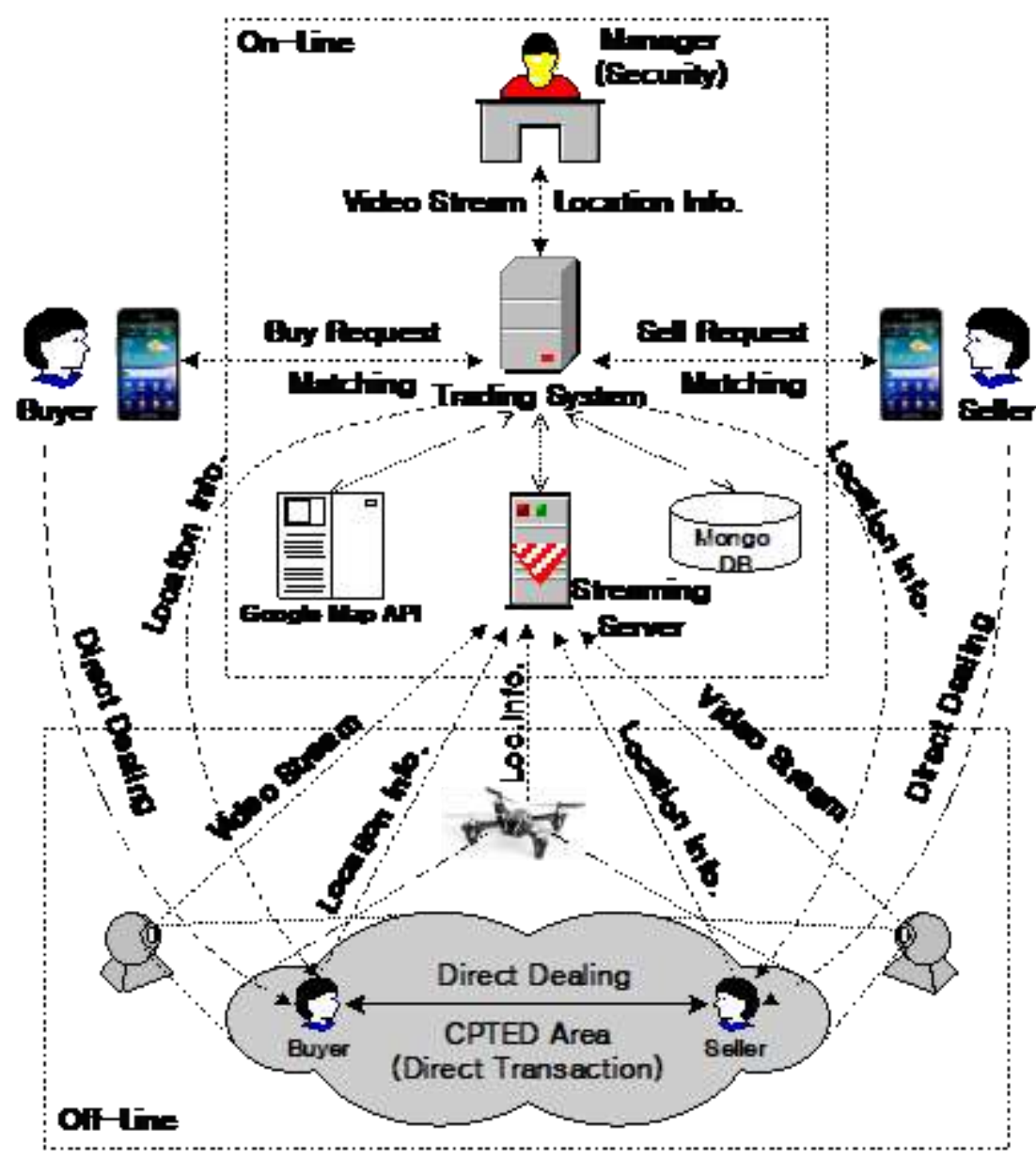

\section{Figure 3. Configuration of the Proposed Platform for Secure Direct Trading Among Individuals}

Third, it has a function that selects offline trading locations. The users can select an agreeable location for the transaction, and they can select only from designated location(s) where CPTED is available.

Fourth, it has a streaming information transmission function. It provides image streams taken by a CCTV at the trading sites so that a manager can monitor the transactions. Direct trading occurs in a limited area where a CCTV, such as in CPTED, is installed.

Fifth, it has a function that provides location information of the seller and buyer. The position information is provided to the transacting parties so that the positions of the traders are displayed in real time on the map.

Sixth, it has a safety transaction-monitoring function. It measures and determines the time parties spend in the trading areas. The manager refers to the measured time data and the CCTV image and monitors whether a safe transaction is being performed.

Seventh, a drone provides trader's location information, destination access information, behavior information, etc. By using this information, we can monitor illegal acts such as violent behavior. And it provides image information from angles and positions that are difficult to monitor with a fixed closed circuit camera. However, this function will be considered in future research because specific development items must be examined in greater detail.

The proposed trading platform among individuals in this research is developed especially for book trading by college students. However, we believe that it can be extended to offline transactions of other items, which can guarantee safe transactions to any consumer or seller. 


\subsection{Development Environment and Architecture of the Proposed Platform}

Table 2 lists the development environment of the proposed platform, and Figure 4 shows the structure of the proposed platform. The proposed platform is configured as a server to store and provide transaction, location, and CCTV streaming information of registered clients. The server uses the Amazon Web Services cloud server, and Node.js is used as the Wowza Media Server and Web server for streaming. MongoDB is used to store information such as video stream and book information. The client information is developed on an Android base, and Retrofit 2 and OKHTTP 3 are used in the communication library. RTSP is used for the streaming service. The streaming information is encoded and transmitted in H.264 format, and the file format used is MP4.

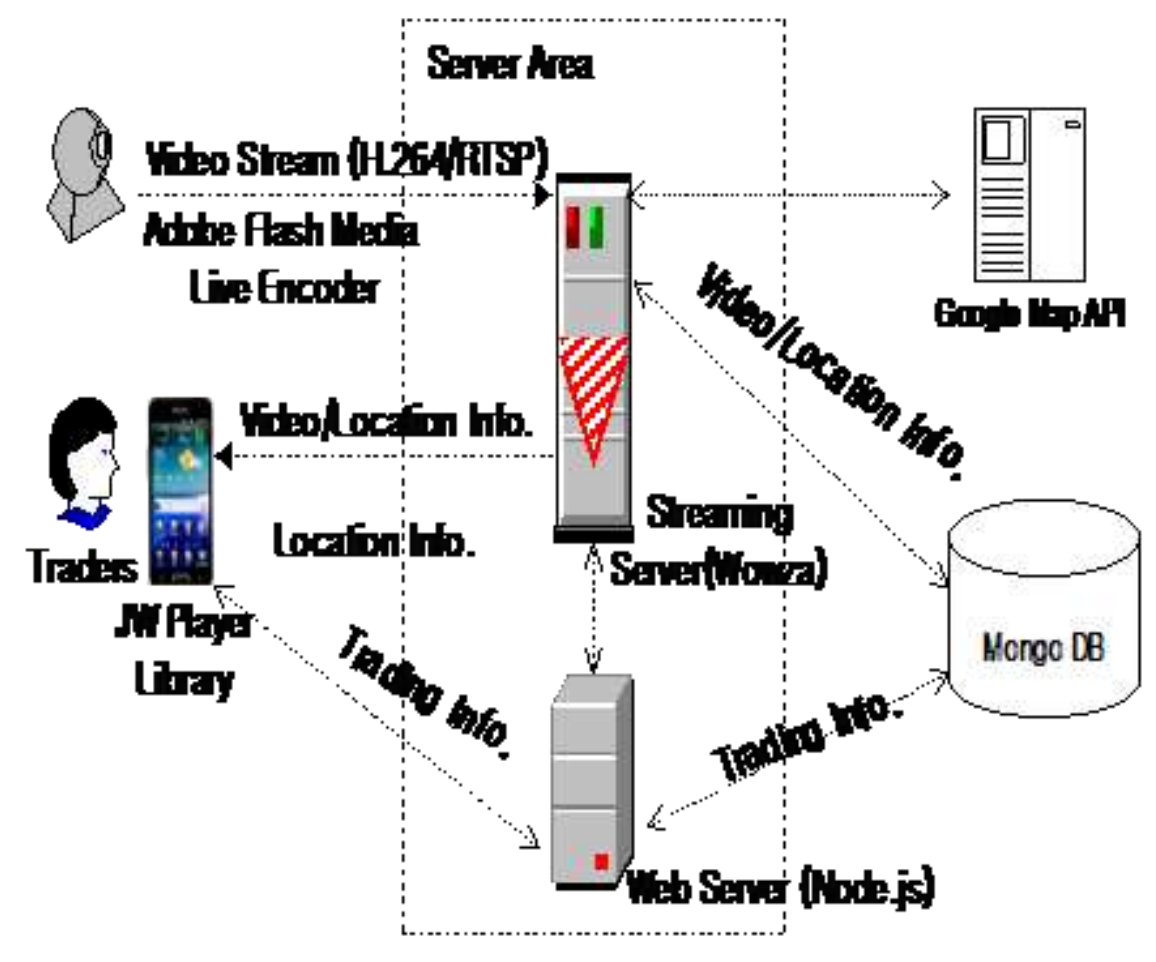

Figure 4. Architecture for Information Transfer in the Proposed Platform

The CCTV uses the H.264 encoding method and Adobe Flash Media Live Encoder for video-stream encoding. The trader mobile client information is developed on an Android base, and the JW Player Library is used to provide a secure stream.

Table 1. Development Environment

\begin{tabular}{|c|c|}
\hline Component & Environments \\
\hline Cloud Server & Amazon Web Service EC2 \\
\hline Server Platform & Wowza Media Server (Media Stream), Node.js (Web Server) \\
\hline OS & Microsoft Windows Server 2012 Base R2 64 bit \\
\hline Client & Android 5.0.1 \\
\hline Database & MongoDB \\
\hline Streaming Tech. & RTSP/Apple HLS \\
\hline Streaming Codec & H.264 \\
\hline Streaming Format & MP4/MOV \\
\hline Positioning Method & Google Map API (GPS / NW signal) \\
\hline $\begin{array}{c}\text { Android N/W } \\
\text { Library }\end{array}$ & Retrofit2, OKHTTP3 \\
\hline
\end{tabular}




\section{Implementation of the Proposed Platform}

Various App user interfaces are developed according to the function of the proposed platform. Figure 5 shows the screens for the login and information on the books for sale. The left side of the figure shows the login screen and the screen for registration by inputting the user name and password. At the time of membership registration, an authentication e-mail is sent to the institution (university) e-mail address, and registration of the user will be approved through e-mail confirmation. This method enhances a user's credentials. It also provides service screens for a user who forgets his user name and password. The two screens on the right of the figure show a list of registered books for sale and detailed information on the books, showing the book cover, title, author, state of the book, and selling price. It also provides a sales opinion from the detailed book information and the trading location.
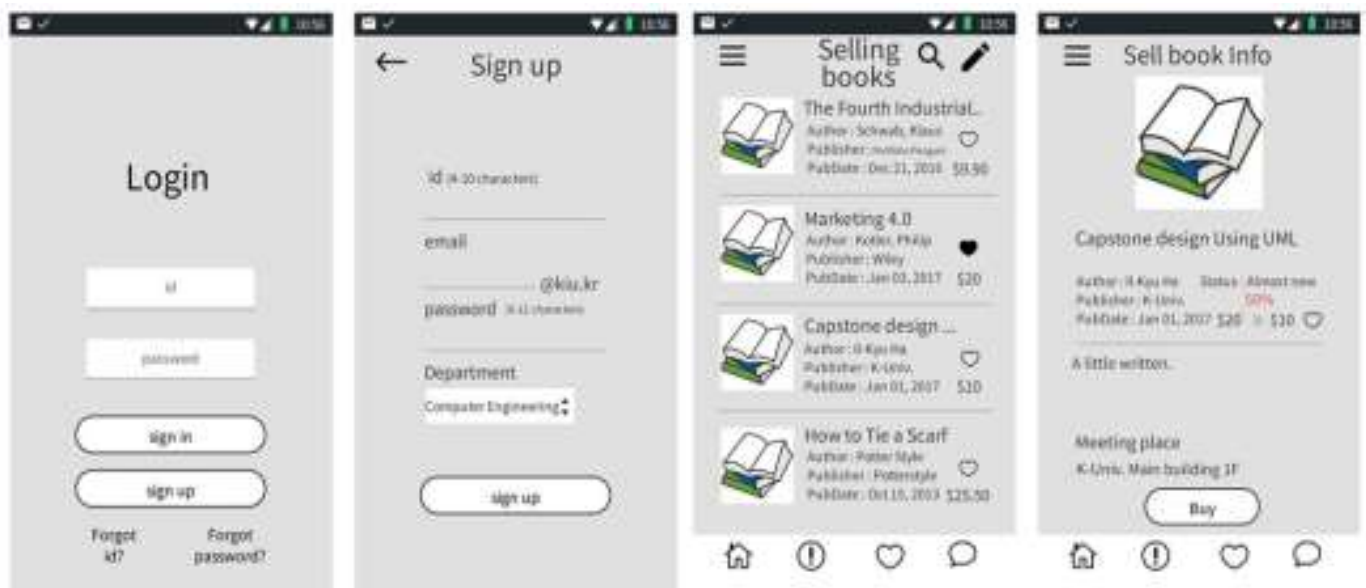

Figure 5. Screens for the Login and Information on the Books for Sale

Figure 6 shows the registration screen of the books for sale, the CCTV streaming information screen, and the screen for the location information. The left screen of the figure is the screen where the user registers the books to be sold.

After entering the title and clicking on the search tab, important information on the book registered in the portal site is automatically displayed. The user can enter the detailed sales information of the book such as the desired price and the state of the book. Interest in the book can be shown by clicking on a heart sign, and pop-up messages provide information when other books by the seller are registered in the future. In the screen, the trading places can be selected; thus, trading places can be selected only in specific places where a CPTED area is available. When a sale is requested, the chat room is activated.

The middle screen of the figure shows the CCTV streaming information. By selecting an item from the CCTV streaming list, a detailed screen of the particular CCTV's view can be displayed. The manager can obtain video and sound information from the location; thus, the transaction can be monitored. The screen on the right shows the locationinformation-sharing screen. The position information is provided to the seller and the buyer via the server. The seller and the buyer can agree on the trading place by sharing their respective locations and can quickly learn who the transacting parties are. A map is provided to the manager and traders showing the distance between the traders and the time to meet at the trading place. 


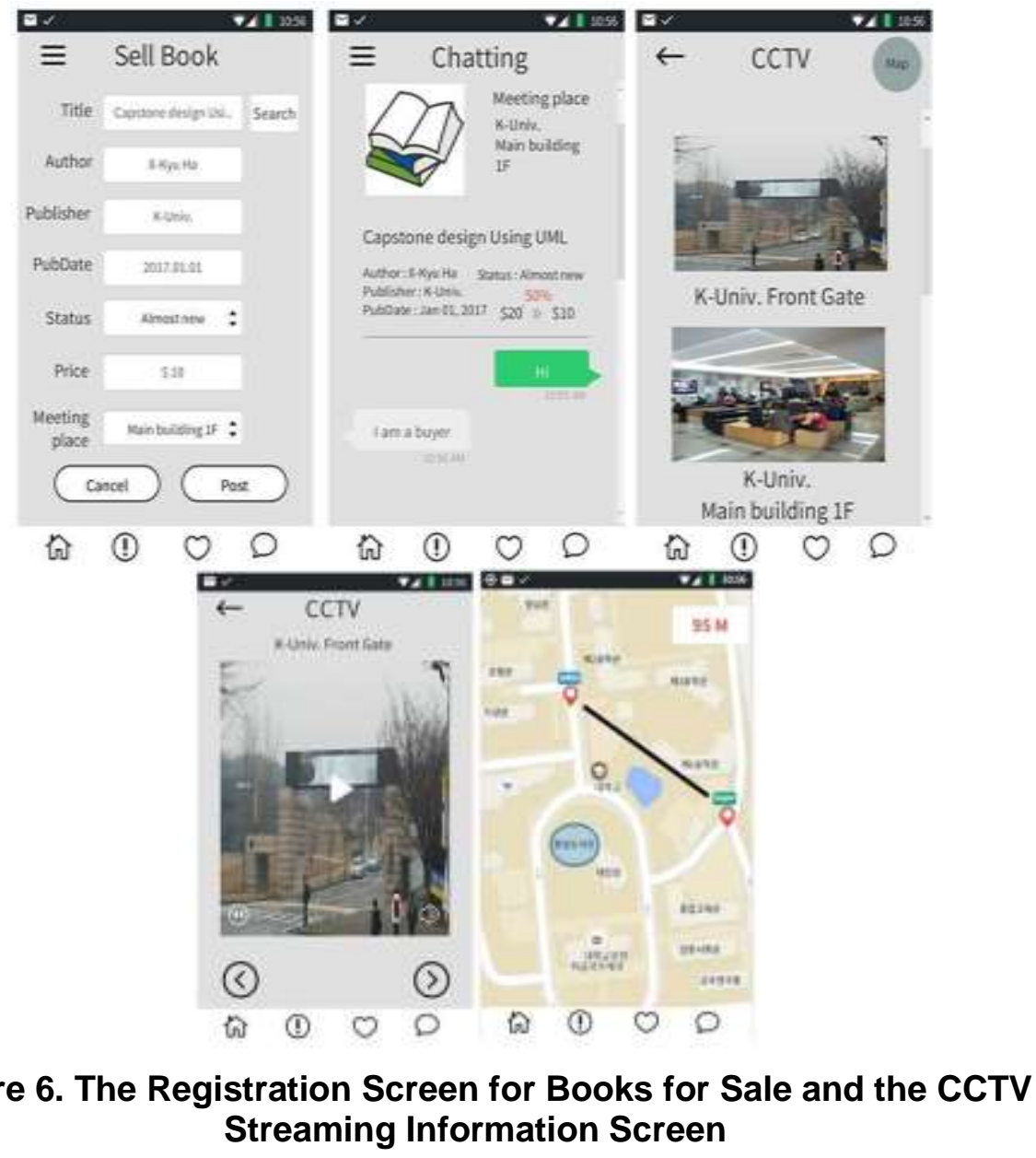

\section{Conclusions}

In this study, we designed and implemented a safe trading platform among individuals that provides transaction location information using drones and CCTV so that a manager can monitor the place of transaction to increase the reliability and safety of direct transactions among individuals. We expect that the proposed platform can provide the following advantages and significance in transactions among individuals.

First, it can potentially contribute to the prevention of fraud by ensuring the safety of the transaction. It can provide CCTV streaming information of the trading place and trader location information to the manager to monitor the transaction. Thus, it can provide secure transactions among individuals and contribute to the prevention of fraud. Second, it can facilitate rapid transactions through the trading system and bring economic benefits to both the seller and buyer. The sellers can quickly dispose of unnecessary books, and the buyers can enjoy the advantage of being able to purchase the necessary books at a lower price than a new book. Third, the proposed trading platform can be extended to various fields. In this research, we designed an application that focused on books. However, the application can be expanded to various other items, e.g., electronic products such as computers, smartphones, and other similar devices. Fourth, the proposed platform is an example of the use of drones in financial transactions. It can serve as an opportunity to expand the use of drones in the financial field in the future, and can be a good model of secure financial transactions using drones.

We believe that the safe inter-individual trading platform proposed in this paper can become a system with high commercialization potential that can be immediately applied to real life. Further, we expect that the platform can be developed into a more 
sophisticated system if some functions such as user discrimination and tracking by applying image-processing technology are added into this platform. In addition, it is expected that the proposed platform will be developed as a more sophisticated secure commerce platform that extends the utilization of drones and acquires various sensing information such as image information as well as location information of traders.

\section{Acknowledgments}

This research was supported by Basic Science Research Program through the National Research Foundation of Korea (NRF) funded by the Ministry of Education (2017R1D1A1B03029895). Lee Young-Min, an undergraduate student, helped to implement the platform. This paper is a revised and expanded version of a paper entitled [Fast Target Information Providing in Secure Direct Dealing System using CCTV and Drones] presented at [Il-Kyu Ha, Jeju, Korea, CRTT 2017, August 22, 2017].

\section{References}

[1] Ministry of Science, ICT and Future Planning and Korea Internet \& Security Agency of Korea, "2016 Internet Usage Survey", available at http://www.msip.go.kr/web/ msipContents/contents.do? mId=MTQ2, (2016).

[2] A. Jo, "A Factorial Analysis of the Influential Factors to the Fear of Crime in Internet Interactions," Master Thesis of Kwangwoon University, (2015).

[3] Seoul Economic Daily, "A copy of a book is also burdened. Book value, five times inflation", available at http://www.sedaily.com/ NewsView/1L2M57R5QQ, (2016).

[4] I. Bekmezci, O. Sahingoz and S. Temel, "Flying Ad-Hoc Networks (FANETs): A survey", Ad Hoc Networks, vol. 11, (2013), pp. 1254-1270.

[5] O. Sahingoz, "Networking Models in Flying Ad-Hoc Networks (FANETs): Concepts and Challenges", Journal of Intelligent Robotic Systems, vol. 74, (2014), pp. 513-527.

[6] S. Waharte and N. Trigoni, "Supporting Search and Rescue Operations with UAVs", Proceedings of the International Conference on Emerging Security Technologies(EST), Canterbury, UK, (2010), September 6-7.

[7] S. Kim, "A critical study on the application of CPTED in Korea”, Korea Criminal Psychology Review, vol. 12 , no. 4, (2016), pp. 47-70.

[8] K. Yoo, "USIM authentication based transaction brokerage system to prevent third party fraud in customer to customer e-commerce environment," Master Thesis of Soongsil University, (2015).

[9] W. Kang, J. Kim, J. Jang, I. Song, and H. Park, "Development of C2C mobile used book trading system," Proceedings of Korea Computer Congress of Korean Institute of Information Scientists and Engineers, Jeju, Korea, vol. 2016, (2016) June 29-July 1, pp. 472-474.

[10] J. Lee, J. Park, S. Cho, Y. Han and Y. Song, "A Design and Implementation of Used Books Trading Web Service using an Auction System”, Journal of Digital Contents Society, vol. 18, no. 1, (2011), pp. 9-16.

[11] D. Kim, "Secure one-time password authentication in mobile environments", Journal of Digital Convergence, vol. 11, no. 12, (2013), pp. 423-430.

[12] E. Park, "Design and Implementation of Smart Care Surveillance System using Location Information," Doctoral Thesis of Kyonggi University, (2014).

[13] Y. Chae, "Analysis and Compensation of RSSI in Wi-Fi Based Indoor Positioning," Master Thesis of University of Seoul, (2010).

[14] M. Kim and K. Jung, "Applicability Analysis of Drone Photogrammetry for Updating Digital Maps," Asia-pacific Journal of Multimedia Services Convergent with Art, Humanities, and Sociology, vol. 6, no. 8, (2016), pp. 603-610.

[15] Y. Park and M. Kim, "Analysis of Characteristics of Ortho Image by Type of Terrain Model using Unmanned Aerial Vehicle," Asia-pacific Journal of Multimedia Services Convergent with Art, Humanities, and Sociology, vol. 5, no. 5, (2015), pp. 505-513.

[16] D. Um and Y. Song, "3D Reality Model Creation and Positioning Accuracy Analysis Using Images Obtained with a Low-cost Rotary Wing Drone," Asia-pacific Journal of Multimedia Services Convergent with Art, Humanities, and Sociology, vol. 6, no. 9, (2016), pp. 235-246.

[17] N. Kumar and S. Jain, "Identification, Modeling and Control of Unmanned Aerial Vehicles," International Journal of Advanced Science and Technology, SERSC Australia, vol. 67, (2014), pp. 1-10.

[18] T. Chung and J. Burdick, "A Decision-Making framework for control strategies in probabilistic search," Proceedings of the 2007 IEEE International Conference on Robotics and Automation, Roma, Italy, (2007) April 10-14. 
[19] A. Symington, S. Waharte, S. J. Julier and N. Trigoni, "Probabilistic target detection by cameraequipped UAVs," Proceedings of the 2010 IEEE International Conference on Robotics and Automation, Anchorage, USA, (2010) May 3-7.

[20] D. Kim, J. Lee, J. Choi and K. Kim, "A POMDP Framework for Dynamic Task Allocation and Reconnaissance of Multiple Unmanned Aerial Vehicles," Journal of KISS, vol. 39, no. 6, (2012), pp. 453-463.

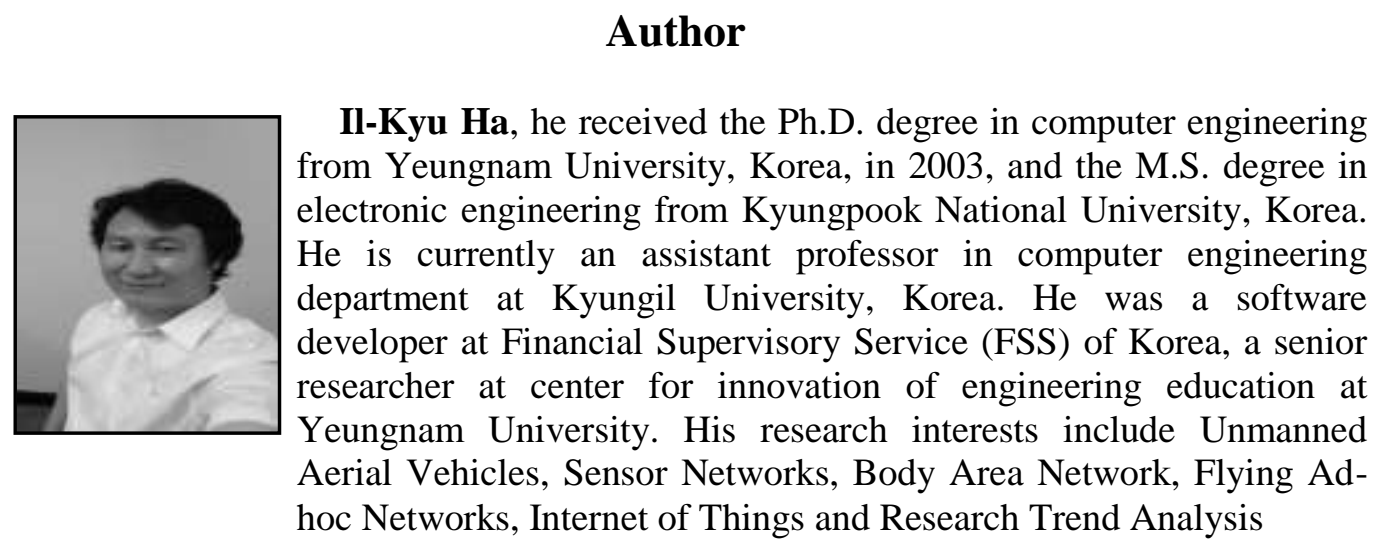

